\title{
Multivariate SPC for total inertial tolerancing
}

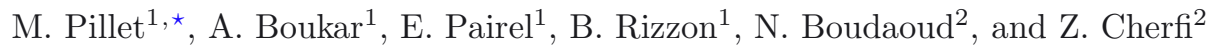 \\ 1 SYMME Laboratory - Université de Savoie, 7 chemin de Bellevue, 74944 Annecy, France \\ 2 ROBERVAL Laboratory, UMR CNRS 6253, Université de Technologie de Compiègne, 60200 Compiègne, France
}

Received: 8 July 2013 / Accepted: 16 July 2013

\begin{abstract}
This paper presents a joint use of the $T^{2}$ chart and total inertial tolerancing for process control. Here, we will show an application of these approaches in the case of the machining of mechanical workpieces using a cutting tool. When a cutting tool in machining impacts different manufactured dimensions of the workpiece, there is a correlation between these parameters when the cutting tool has maladjustment due to bad settings. Thanks to total inertial steering, the correlation structure is known. This paper shows how $T^{2}$ charts allow one to take this correlation into account when detecting the maladjustment of the cutting tool. Then the total inertial steering approach allows one to calculate the value of tool offsets in order to correct this maladjustment. We will present this approach using a simple theoretical example for ease of explanation.
\end{abstract}

Keywords: Machining; adjustment; $T^{2}$; multivariate; inertial tolerancing

\section{Introduction}

The works presented in this paper are based on total inertial tolerancing proposed by Pillet $[1,2]$. The objective of this paper is to propose a method to steer a machining process by minimizing the inertia of the surfaces. The proposed method is based on multivariate SPC.

For a given surface, the inertia is calculated using the vector of the deviations between the theoretical position of the surface and the actual position. Its calculation therefore requires several measured points on the surface and their deviations according the normal to the surface. The inertia $^{1}$ of a surface is calculated using the following relationship (Eq. (1)).

$$
I_{i}=\sqrt{\frac{1}{n} \sum_{j=1}^{n}\left(X_{i j}-\tau_{j}\right)^{2}}=\sqrt{\sigma_{i}^{2}+\left(\overline{X_{i j}-\tau_{j}}\right)^{2}} .
$$

With

$X_{i j}$ : point $j$ measured on the surface $i$;

$\sigma_{\mathrm{i}}$ : standard deviation of the points measured on the surface $i$;

$\tau_{j}$ : target point $j$ measured relative to the datum system of the part;

$n$ : number of points measured on the surface;

$I_{i}$ : surface inertia.

Pillet [1] and Adragna et al. [3] show that this measure of variability around the target provides a better repre-

\footnotetext{
* Correspondence: maurice.pillet@univ-savoie.fr

1 Inertial tolerances are defined by the French standard XP E04-008 (2009).
}

sentation of statistical behavior during assembly than the conventional zone specification.

The principle behind total inertial steering [4] is to establish a direct link between the parameter settings available on the machine (mainly tools offset) and the position of the points in a coordinate system associated with the machine.

It uses all the available information (the deviation on each measured point) directly. There is no "lost information" induced by the passage through a classic tolerancing method by lengths, diameters, angles, etc. This advantage allows one to obtain a level of accuracy superior to conventional approaches. The Total Inertial Steering approach minimizes the mean square deviations. By the calculating using all the measured points, total inertial steering is by its very nature - a multidimensional approach.

Statistical process control has long been interested in the control of multidimensional processes. The best known approach is the one proposed by Hotelling which calculates the statistic $T^{2}$ [5]. This approach has been extensively commented on $[6,7]$, and several improvements have been put forward. Ghute and Shirke [8] have presented a multivariate synthetic control chart consisting of two subcharts: a $T^{2}$ sub-chart and a CRL (conforming run length) sub-chart. The CRL sub-chart improves the ARL (average run length). Champ and Aparisi [9] have proposed two double sampling (Hotelling's $T^{2}$ charts). Aparisi and Deuna [10] have developed the synthetic $T^{2}$ control chart, which is compared to other control charts. It is shown that it performs consistently better than the $T^{2}$ chart. Boudaoud and Cherfi [11] propose a new statistic for monitoring multivariate trend processes. They focus on 


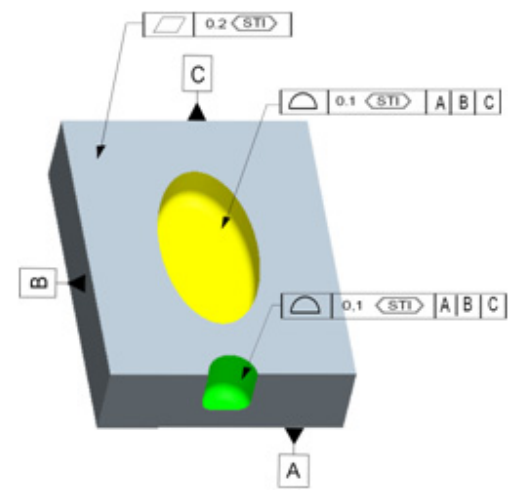

Fig. 1. Inertial specification of the part. (\$T): Symbol of inertial tolerancing.

the choices of more sensitive statistics than the classical Hotelling $T^{2}$ statistic. The improvement is significant in the case of processes where incipient trends are considered.

This paper focuses on the interest of the Hotelling $T^{2}$ chart in the total inertial tolerancing environment. The originality of this paper is to use the power of inertial steering which allows one to calculate an incidence matrix using the link between the tools offsets and their characteristics, in association with one Hotelling chart per tool offset in order to use the multidimensional information of the incidence matrix.

\section{Total inertial steering (TIS)}

\subsection{Example}

The objective of any production process is to manufacture parts that conform to the requirements of the geometric specifications established by a CAD system (computeraided design). This requirement is materialized by a digital target that we specify using an acceptable level of variability (tolerances). As with any production processes that induce dispersions, the steering of machines is necessary to satisfy the required level of variability. The TIS approach is a tool that is able to reconcile the real workpiece to its digital model through the measured points on all the surfaces of the workpiece. Inertia is the quality indicator of the surface in inertial steering. Pillet [1] showed that mastering this inertia, allows us to control the process, because the inertia (Eq. (1)) contains both the information concerning the dispersion and the decentering.

Figure 1 shows a drawing of the finished part which is specified in inertial tolerancing. This example reminds us of the principle of inertial tolerancing.

We machine a block of rectangular material (dimensions $25 \mathrm{~mm} \times 20 \mathrm{~mm}$ ) on a CNC milling machine, which is fixed to the milling machine table. Three stops are used to position the slug on this table. A clamping system ensures it will not move during the various operations (see Fig. 2).

We make an elliptical pocket in the slug by contour milling and create a notch using the same tool, which is

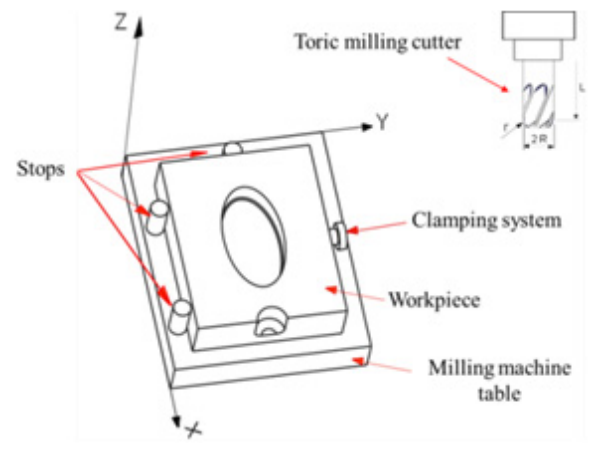

Fig. 2. Defining of action axis of the tool - machining assembly.

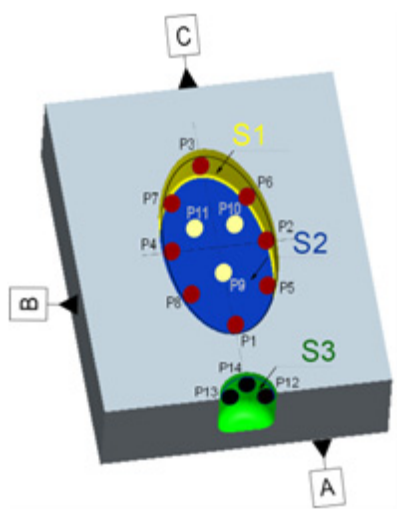

Fig. 3. Measured points on the workpiece.

a toric milling cutter. The elliptical pocket and the notch have the same inertial tolerance as shown in Figure 1.

We decided randomly to measure eleven points on the elliptical pocket (eight on its side (S1) and three on the bottom (S2) and three points on the notch S3 to set the tool (see Fig. 3). The surfaces of the fillets generated by the radius at the end of the milling cutter and the bottom of the notch are not probed. If the fillets are not the right shape, the tool will be sharpened or replaced. The points on the notch are measured on its cylindrical portion to allow any repositioning of the notch relative to the ellipse. Figure 3 shows the measured points on the workpiece.

Table 1 gives the coordinates of the points and of the normals vector expressed in the frame of reference of the part, and the deviations of these points along the local normal vectors. The objective of total inertial steering is to minimize the inertia of these deviations.

\subsection{Incidence matrix}

Surfaces S1, S2 and S3 are generated using the same cutting tool. This cutting tool can be adjusted by acting on its tool length offset $(L)$ along the $Z$-axis and its tool radius offset $(R)$. Parameters $T x, T y$ and $R z$ are also corrected to enable any necessary repositioning of the shapes on their targets. The program variables of displacement are also used to rebalance the program relative to the workpiece. Parameters $L$ and $R$ are the dimensional 
Table 1. Expression of the points in the reference part.

\begin{tabular}{cccccccccc}
\hline Surface & Inertial tolerance & Point & $X$ & $Y$ & $Z$ & $N x$ & $N y$ & $N z$ & $e(n i)=\bar{X}_{i}$ \\
\hline \multirow{5}{*}{ S1 } & P1 & 20 & 10 & 5 & -1 & 0 & 0 & 0.43 \\
& & P2 & 12.5 & 14 & 5 & 0 & -1 & 0 & -0.61 \\
& & P3 & 5 & 10 & 5 & 1 & 0 & 0 & -0.44 \\
& \multirow{4}{*}{0.1} & P4 & 12.5 & 6 & 5 & 0 & 1 & 0 & 0.30 \\
& & P5 & 17.8 & 12.83 & 5 & -0.47 & -0.88 & 0 & -0.34 \\
& & P6 & 7.2 & 12.83 & 5 & 0.47 & -0.88 & 0 & -0.34 \\
& & P7 & 7.2 & 7.17 & 5 & -0.47 & 0.88 & 0 & 0.25 \\
& & P8 & 17.8 & 7.17 & 5 & 0.47 & 0.88 & 0 & 0.26 \\
\hline \multirow{3}{*}{ S2 } & \multirow{3}{*}{0.1} & P9 & 15 & 10 & 3 & 0 & 0 & 1 & -0.01 \\
& & P10 & 10 & 12.5 & 3 & 0 & 0 & 1 & 0.01 \\
& & P11 & 10 & 7.5 & 3 & 0 & 0 & 1 & -0.03 \\
\hline \multirow{3}{*}{ S3 } & \multirow{3}{*}{0.1} & P12 & 24 & 12 & 4 & -0.55 & -0.835 & 0 & -0.37 \\
& & P13 & 24 & 8 & 4 & -0.55 & 0.835 & 0 & 0.56 \\
& & P14 & 22.5 & 10 & 4 & 1 & 0 & 0 & -0.27 \\
\hline
\end{tabular}

parameters. They are used to modify the dimension of the workpiece.

The displacement of each point can be calculated using the method of small displacements [12] so, by assuming the use of the small displacements methods in relation to the curvatures of the surface, it is possible to linearize the deviation to the point $P_{i}$ with respect to its target surface towards its displacement, according to the equation (2):

$$
e i=\xi i+a i T x+b i T y+N i R z+c i L+R
$$

With:

$\xi i$ : initial deviations compared to the target points;

$e i$ : final deviations after correction;

$L$ : tool length offset;

$R$ : tool radius offset;

$T x: X$ offset;

Ty: $Y$ offset;

$R z: Z$ rotation;

$a i, b i, c i$ : direction cosines of the normal $\overrightarrow{n i}$ to the target surface.

$N i$ : components on the $X$-axis of the vector $\overrightarrow{O P} i \wedge \overrightarrow{n i}$.

If there are $n$ points on the surface carried by the tool, we obtain a system of $n$ equations where the variables are the parameters of the movement of the tool and which can be written in the following matrix form (Eq. (3)):

$$
[a](C)+(-e)=-(\xi)
$$

where $C$ is the vector of correctors.

The matrix $[a]$ is called the incidence matrix because it contains the influence coefficients of the corrections $L$, $R, T x, T y$ and $R z$ on the deviations of the points. The incidence matrix is given in Table 2 .

\subsection{Steering matrix}

The originality of the method proposed is to calculate a $T^{2}$ chart for each tool offset. The $T^{2}$ chart is built using the non-null term of the incidence matrix for the column associated with the tool offset. If the tool is not on the
Table 2. Incidence matrix calculated in the reference machine.

\begin{tabular}{cccccc}
\hline \multicolumn{5}{c}{ Tool offsets } \\
\hline Point & $L$ & $R$ & $T x$ & Ty & $R z$ \\
\hline P1 & 0 & 1 & -1 & 0 & 10 \\
P2 & 0 & 1 & 0 & -1 & -12.5 \\
P3 & 0 & 1 & 1 & 0 & -10 \\
P4 & 0 & 1 & 0 & 1 & 12.5 \\
P5 & 0 & 1 & -0.47 & -0.88 & -9.63 \\
P6 & 0 & 1 & 0.47 & -0.88 & -12.37 \\
P7 & 0 & 1 & -0.47 & 0.88 & 9.7 \\
P8 & 0 & 1 & 0.47 & 0.88 & 12.3 \\
P9 & 1 & 0 & 0 & 0 & 0 \\
P10 & 1 & 0 & 0 & 0 & 0 \\
P11 & 1 & 0 & 0 & 0 & 0 \\
P12 & 0 & 1 & -0.55 & -0.835 & -13.44 \\
P13 & 0 & 1 & -0.55 & 0.835 & 24.44 \\
P14 & 0 & 1 & 1 & 0 & -10 \\
\hline
\end{tabular}

target, all the characteristics concerned by the tool are probably decentered. The incidence matrix includes this information and the weight of the tool's impact. Thanks to this way of proceeding, the $T^{2}$ chart reduced to the implied characteristics is an excellent way to detect a deviation.

The objective of setting the machine is to bring the points on their target positions. It is necessary to calculate the displacement of the tool, i.e. the parameters $T x, T y$, $R z, L$ and $R$ that minimize the sum of the squares of the new deviations $e i$. This type of calculation, called multiple linear regression, consists in multiplying the matrix of the initial deviations $(\xi i)$ by the well-known Gauss pseudoinverse matrix $\left[a^{*}\right]$ of the incidence matrix (see Eq. (4)):

$$
(C)=\left[a^{*}\right](\xi) \text { with }\left[a^{*}\right]=\left([a]^{T}[a]\right)^{-1}[a]^{T} \text {. }
$$

By minimizing the sum of the squared deviations, we find the corrections which minimize the inertia of the surface calculated by the equation (1) immediately. The matrix $\left[a^{*}\right]$ is called the steering matrix. It is given in Table 3 . 
Table 3. Steering matrix $\left[a^{*}\right]$.

\begin{tabular}{ccccccccccccccc}
\hline & \multicolumn{10}{c}{} & \multicolumn{10}{c}{ Point } \\
\hline Tool offsets & P1 & P2 & P3 & P4 & P5 & P6 & P7 & P8 & P9 & P10 & P11 & P12 & P13 & P14 \\
\hline$L$ & 0 & 0 & 0 & 0 & 0 & 0 & 0 & 0 & 0.33 & 0.33 & 0.33 & 0 & 0 & 0 \\
$R$ & 0.09 & 0.09 & 0.09 & 0.09 & 0.09 & 0.09 & 0.09 & 0.09 & 0 & 0 & 0 & 0.09 & 0.09 & 0.09 \\
$T x$ & -0.14 & 0.09 & 0.14 & -0.09 & -0.12 & 0.29 & -0.4 & 0.24 & 0 & 0 & 0 & -0.36 & 0.21 & 0.14 \\
$T y$ & -0.15 & -0.32 & 0.15 & 0.32 & -0.1 & -0.47 & 0.68 & -0.11 & 0 & 0 & 0 & 0.3 & -0.47 & 0.15 \\
$R z$ & 0.01 & 0.01 & -0.01 & -0.01 & 0 & 0.02 & -0.04 & 0.02 & 0 & 0 & 0 & -0.03 & 0.04 & -0.01 \\
\hline
\end{tabular}

\section{The in/off control in TIS}

\subsection{The in/off control in TIS}

The principle behind statistical process control is to dissociate two situations: a process that is "under control" from one that is "out of control". How can these two situations be separated? How can we determine if the deviations measured on the surfaces are the expression of random variations or if these deviations require us to intervene as in the settings?

The monitoring process using control charts is traditionally described in two phases.

Phase I: Control charts are used to test retrospectively whether or not the process was in control when the first subgroups were measured.

Phase II: Control charts are used for testing whether the process remains in control when future subgroups will be measured.

In TIS, these two phases use different controls charts:

Phase I: Shewhart charts are used to identify the short term standard deviation for each point. Montgomery [13] and Pillet [14] describe this phase.

Phase II: A $T^{2}$ chart is used for testing whether the process remains in control. All the improvements proposed for $T^{2}$ charts that were presented in the literature review can be used. Only the basic $T^{2}$ chart is presented in this paper.

\subsection{The $T^{2}$ chart}

For each measured point, the short-term deviation is calculated. There may be significant differences between the short-term standard deviations. To deal with this kind of data, the multidimensional analysis method chosen must take into account the variability in each different direction. The $T^{2}$ chart method is well-suited for such calculations.

Assuming that the vector $x$ follows a $p$-dimensional normal distribution, denoted as $N p\left(\mu_{0}, \Sigma_{0}\right)$, that there are $m$ samples each of size $n \geqslant 1$ available from the process and that the vector observations $X$ are not time dependent, a control chart can be based on the sequence of the following statistic (Eq. (5)):

$$
D_{i}^{2}=n\left(\bar{X}_{i}-\mu_{0}\right)^{t} \Sigma_{0}^{-1}\left(\bar{X}_{i}-\mu_{0}\right)
$$

where

$n$ : the sample size;

$\bar{X}_{i}$ : the vector of the sample averages of the $i$ th rational subgroup;

$\mu_{0}$ : the known vector of means;

$\Sigma_{0}$ : the known variance-covariance matrix.

The $D_{i}^{2}$ statistic represents the weighted distance (Mahalanobis distance) of any point from the target $\mu_{0}$. The $D_{i}^{2}$ statistic follows a $\chi^{2}$-distribution with $p$ degrees of freedom.

If $\mu_{0}$ is replaced by $\overline{\bar{X}}_{0}$, and $\Sigma_{0}$ is replaced by $[\bar{S}]$, and $\bar{X}_{i}$ is the mean of the $i$ th rational subgroup then, according to Ryan [15], the $D_{i}^{2} / c_{0}(p, m, n)$ statistic follows an $F$-distribution with $p$ and $(m n-m-p+1)$ degrees of freedom.

where:

$$
C o(p, m, n)=[p(m-1)(n-1)](m n-m-p+1)
$$

$\overline{\bar{X}}_{0}$ : the overall sample mean vector;

$[\bar{S}]$ : the pooled sample variance-covariance matrix

$\left(\bar{S}_{i i}=\left(\overline{R_{i}} / d_{2}\right)^{2}\right.$;

$\bar{R}$ : moving range;

$d_{2}$ : constant from the range distribution.

Thus, a multivariate Shewhart control chart for the process mean, with unknown parameters, is based on the following statistical relation (Eq. (6)):

$$
T_{i}^{2}=n\left(\bar{X}_{i}-\overline{\bar{X}}_{0}\right)^{t}\left[\bar{S}^{-1}\right]\left(\bar{X}_{i}-\overline{\bar{X}}_{0}\right) .
$$

The control limit in phase II is:

$$
L u=\frac{p(m+1)(n-1)}{n m-m-p+1} F_{\alpha}, p, n m-m-p+1 .
$$

\subsection{Adaptated $T^{2}$ chart for TIS - phase I}

In TIS, phase I is used to calculate the pooled sample variance for each point $\overline{S_{1}}$ and calculate the $\overline{\bar{X}}_{0}$ vector. The pooled sample is calculated using the traditional Shewhart chart. Table 4 shows the variance for the example.

Assuming that:

1. When the process is in control the variation on each point is purely random.

2. The correlation between different points is the consequence of a decentering of a tool offset.

3. $\overline{\bar{X}}_{0}$ Represents the best adjustment possible with the Tool offset. 
Table 4. Result of phase I in the example.

\begin{tabular}{ccccc}
\hline Surface & Inertial tolerance & Point & $\overline{\bar{X}}_{0}$ & $\overline{S_{1}}$ \\
\hline \multirow{5}{*}{ S1 } & & P1 & -0.0120 & 0.0705 \\
& & P2 & -0.0312 & 0.0708 \\
& & P3 & 0.0139 & 0.0703 \\
& \multirow{3}{*}{0.1} & P4 & 0.0212 & 0.0703 \\
& & P5 & -0.0273 & 0.0686 \\
& & P6 & 0.0429 & 0.0686 \\
& & P7 & -0.0068 & 0.0702 \\
S2 & \multirow{3}{*}{0.1} & P8 & -0.0193 & 0.0705 \\
\hline \multirow{3}{*}{ S3 } & & P9 & 0.0372 & 0.071 \\
& & P10 & 0.0231 & 0.0692 \\
& \multirow{3}{*}{0.1} & P1 & -0.0603 & 0.0712 \\
\hline
\end{tabular}

Table 5. Variance matrix $S_{T Y}$.

\begin{tabular}{lcccccccc}
\hline \multicolumn{7}{c}{ Variance matrix $S_{T Y}$} \\
\hline & P2 & P4 & P5 & P6 & P7 & P8 & P12 & P13 \\
\hline P2 & 0.005 & & & & & & & \\
P4 & & 0.0049 & 0 & 0 & 0 & 0 & 0 & 0 \\
P5 & & 0.0047 & 0 & 0 & 0 & 0 & 0 \\
P6 & & & 0.0047 & 0 & 0 & 0 & 0 \\
P7 & & & & 0.0049 & 0 & 0 & 0 \\
P8 & & & & & 0.005 & 0 & 0 \\
P12 & & & & & & 0.0051 & 0 \\
P13 & & & & & & & 0.0049 \\
\hline
\end{tabular}

we will suppose that the $[\bar{S}$ matrix is diagonal. The appearance of a correlation structure is a symptom of the need to adjust a tool offset.

For each tool offset $j$, it is possible to identify the variance matrix $\left[S_{T O j}\right]$ from the incidence matrix $[a]$ and the $\overline{S_{1}}$ vector. The null column and row are removed

$$
\left[S_{T O j}\right]=\left[\begin{array}{ccccc}
\bar{S}_{1}^{2} & 0 & 0 & 0 & 0 \\
0 & \ldots & 0 & 0 & 0 \\
0 & 0 & \bar{S}_{i}^{2} & 0 & 0 \\
0 & 0 & 0 & \ldots & 0 \\
0 & 0 & 0 & 0 & \bar{S}_{1 n}^{2}
\end{array}\right]
$$

For the $T_{y}$ tool offset, the variance matrix is given in Table 5.

Ideally, $\overline{\bar{X}}_{0}$ is a null vector. It means that all the points are in the exact theoretical position. However, some deviation cannot be eliminated with the tools offset available Some deviation cannot be corrected by tool offset (such as metal deformation for example). Then a null vector increases the $T^{2}$ value because the deviations vector includes deformation that is impossible to correct.

$\overline{\bar{X}}_{0}$ is calculated using the following relation (Eq. (7)), which stems from the possible best fit:

$$
\overline{\bar{X}}_{0}=\overline{(\xi)-[a](C)}=\bar{e}
$$

i.e. the average residue after adjustment in phase I. Table 4 shows the vector $\overline{\bar{X}}_{0}$ for the example.

\begin{tabular}{|c|c|c|c|c|c|c|}
\hline & & & & $\overline{\bar{X}}_{i}-\overline{\bar{X}}$ & & \\
\hline Surface & Point & 1 & 2 & 3 & 4 & 5 \\
\hline & P2 & 0.031 & 0.136 & -0.030 & -0.045 & -0.163 \\
\hline & P4 & -0.088 & 0.038 & 0.090 & 0.031 & 0.099 \\
\hline & P5 & 0.107 & 0.055 & -0.025 & -0.184 & -0.033 \\
\hline S1 & P6 & -0.024 & -0.125 & -0.079 & -0.166 & -0.293 \\
\hline & P7 & 0.051 & 0.013 & -0.053 & -0.038 & 0.117 \\
\hline & P8 & 0.139 & 0.099 & 0.021 & 0.100 & 0.151 \\
\hline & $\mathrm{P} 12$ & -0.147 & -0.056 & -0.098 & -0.117 & -0.123 \\
\hline S3 & P13 & -0.051 & 0.062 & 0.180 & 0.149 & 0.155 \\
\hline & $T^{2}$ & 26.99 & 22.72 & 24.74 & 46.17 & 81.81 \\
\hline
\end{tabular}

Table 6. Example for the $T^{2}$ calculation for the $T_{y}$ tool offset.

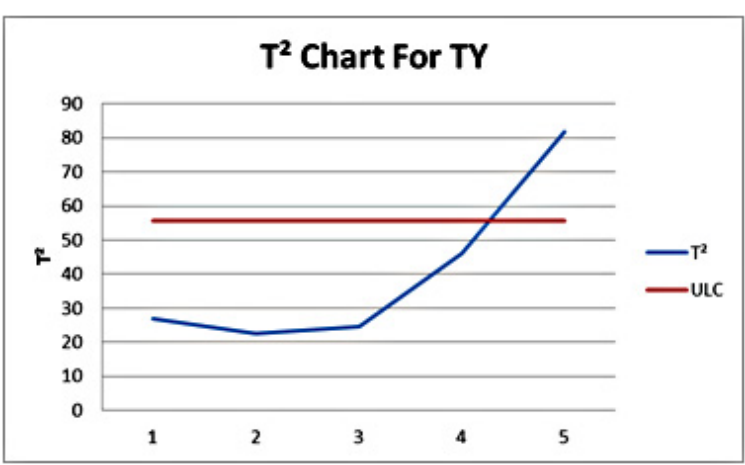

Fig. 4. Measured point on the workpiece.

Table 7. Vector $\bar{X}_{i}-5$ th sample.

\begin{tabular}{ccccccc}
\hline \multicolumn{7}{c}{ Vector $\bar{X}_{i}-\overline{\bar{X}}_{0}-5$ th sample } \\
\hline Point 1 & Point 2 & Point 3 & Point 4 & Point 5 & Point 6 & Point 7 \\
\hline 0.035 & -0.163 & -0.083 & 0.099 & -0.033 & -0.293 & 0.117 \\
\hline Point 8 & Point 9 & Point 10 & Point 11 & Point 12 & Point 13 & Point 14 \\
\hline 0.151 & -0.022 & -0.043 & 0.084 & -0.123 & 0.155 & -0.028 \\
\hline
\end{tabular}

Table 8. Example for the $T^{2}$ calculation.

\begin{tabular}{cccccc}
\hline Offset & $L$ & $R$ & $T x$ & $T y$ & $R z$ \\
\hline$p$ & 3 & 11 & 9 & 8 & 11 \\
$L u$ & 21.53 & 93.6 & 66.0 & 55.7 & 93.6 \\
$T^{2}$ & 3.75 & 85.32 & 70.82 & 81.81 & 85.32 \\
Situation & OK & OK & KO & KO & OK \\
\hline
\end{tabular}

\subsection{Adapted $T^{2}$ chart for TIS - phase II}

In phase II, for each samples $i$ and each tool offset $j$, the $T_{i j}^{2}$ statistic is calculated by equation (6). If the $T_{i j}^{2}$ is upper $L u$, an "off control" situation is detected and a correction is calculated by equation (4). This calculation is illustrated from the mean vector for the $T_{y}$ tool offset in Table 6 .

With the data in Table 6 , where $\alpha=0.0027, n=2$, $p=8$ and $m=25$, the $L u$ limit is 55.66. The $T^{2}$ chart is given in Figure 4.

The 5th sample gives a $T^{2}$ upper than the $L u$ limit, an adjustment on the $T y$ is necessary.

Same calculations are made on the other tool offset. For the 4 th sample (Tab. 7 ), the $T^{2}$ for each tool offset is presented in Table 8. 
Table 9. Steering matrix $\left[a^{*}\right]$ reduce for $T_{x} T_{y}$.

\begin{tabular}{ccccccccccccccc}
\hline & \multicolumn{11}{c}{ Point } \\
\hline Tool offsets & P1 & P2 & P3 & P4 & P5 & P6 & P7 & P8 & P9 & P10 & P11 & P12 & P13 & P14 \\
\hline Tx & -0.14 & 0.09 & 0.14 & -0.09 & -0.12 & 0.29 & -0.4 & 0.24 & 0 & 0 & 0 & -0.36 & 0.21 & 0.14 \\
Ty & -0.15 & -0.32 & 0.15 & 0.32 & -0.1 & -0.47 & 0.68 & -0.11 & 0 & 0 & 0 & 0.3 & -0.47 & 0.15 \\
\hline
\end{tabular}

Table 10. $T_{x}$ and $T_{y}$ value.

\begin{tabular}{cc}
\hline Tool offset & Adjustment \\
\hline$T_{x}$ & 0.48 \\
$T_{y}$ & -0.159 \\
\hline
\end{tabular}

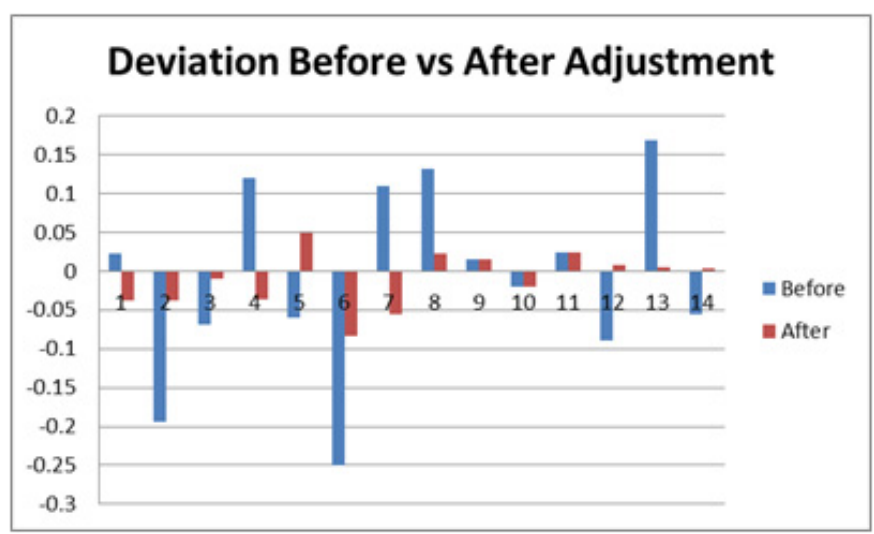

Fig. 5. Deviations before and after adjustment.

The tool offset adjustment is calculated by equation (4) from the vector $\xi i$ and the matrix $a^{*}$ reduced to the "off control" tool offset $\left(T_{x}, T_{y}\right)$.

The best fit is given by equation (4).

Table 10 gives the values of the $T x$ and $T y$ tool offsets calculated from the Table 9.

The expected situation after adjustment is given by equation (7). Figure 5 shows the adjustment for each point.

\section{Discussion and conclusion}

Total inertial steering brings a solution to carry out statistical process control without a parameterization by lengths, diameters, angles, etc. The information used is the root information: the deviation from the target surface. The paper shows the way to calculate the best adjustment directly from the root information, and to identify the situations needing adjustment thanks to the use of a $T^{2}$ chart.

In example used in this paper, 14 points are measured on the workpiece. In real-life cases, the number of measured points runs into hundreds or even, thousands. It is impossible to use an individual control chart for each point. With the $T^{2}$ control chart, the number of control charts is limited and equal to the number of tools offset. Each control chart is calculated using a high quantity of data. Thus, the precision of the steering is very high.

The process described in this paper needs to use a very large matrix with dynamic calculations of an $a^{*}$ matrix. However, even if the dimensions of the matrix are expressed in thousands of lines, the calculation is instantaneous with modern-day computers.

$T^{2}$ control charts use incidence matrix information. This matrix gives the correlation structure which can be used to detect maladjustment. By using the raw information for the deviation and the expected correlation structure given by the incidence matrix, Total Inertial Steering and $T^{2}$ charts offer a very efficient method to guarantee very high quality in $3 \mathrm{D}$ workpiece machining.

Many improvements in the method presented could be made, beginning with looking at the different possible evolutions of the $T^{2}$ chart in a TIS environment.

\section{References}

1. M. Pillet, Improving the productivity and industrial deployment of Inertial Tolerancing - Améliorer la productivité, déploiement industriel du tolérancement inertiel (Eyrolles, Éditions d'Organisation, 2010)

2. M. Pillet, Inertial tolerancing in the case of assembled products. Recent advances in integrated design and manufacturing in mechanical engineering, pp. 85-94, ISBN 14020-1163-6

3. P.A. Adragna, M. Pillet, S. Samper, F. Formosa, Guaranteeing a maximum Non-Conformity Rate on the assembly resulting from a statistical tolerancing approach, Computer Aided Tolerancing (CAT), Erlangen, Germany, 2007

4. D. Denimal, M. Pillet, The Adjustment and Monitoring of Freeform Surfaces using Inertial Tolerancing, ASIGURAREA CALITATII - QUALITY ASSURANCE XVII, 8-16 (2011)

5. H. Hotelling, The generalization of Student's ratio, Ann. Math. Stat. 2, 360-378 (1931)

6. S. Bersimis, S. Psarakis, J. Panaretos, Multivariate Statistical Process Control Charts: An Overview, Qual. Reliab. Eng. Int. 23, 517-543 (2007)

7. R.L. Mason, J.C. Young, Multivariate Statistical Process Control with Industrial Application, ASA-SIAM Series on Statistics and Applied Probability (Copyright ${ }^{\circledR} 2002$ by the American Statistical Association and the Society for Industrial and Applied Mathematics, 2002) 
8. V.B. Ghute, D.T. Shirke, A multivariate synthetic control chart for monitoring process mean vector, Commun. Stat. Theory Meth. 37, 2136-2148 (2008)

9. C.W. Champ, F. Aparisi, Double sampling Hotelling's $T^{2}$ charts, Qual. Reliab. Eng. Int. 24, 153-166 (2008)

10. F. Aparisi, M.A. Deuna, The design and performance of the multivariate synthetic $T^{2}$ control chart, Commun Stat. Theory Meth. 38, 173-192 (2009)

11. N. Boudaoud, Z. Cherfi, A comparative study of cusum and EWMA charts: detection of incipient drifts in a multivariate framework, Qual. Eng. 17, 703-709 (2004)
12. P. Bourdet, A. Clement, A study of optimal - criteria identification based on the small - displacement screw model, CIRP Ann. 37, 503-506 (1988)

13. D.C. Montgomery, Introduction to statistical quality control, 4th edn. (Wiley, 2001)

14. M. Pillet, Applying Statistical Process Control (Appliquer la Maîtrise Statistique des processus MSP/SPC) (Eyrolles, Éditions d'Organisation, 2008)

15. T.P. Ryan, Statistical Methods for Quality Improvement (Wiley, New York, 2000) 